\title{
A Preliminary Study of the Calibration for the Rotating Fan-Beam Scatterometer on CFOSAT
}

\author{
Jintai Zhu, Xiaolong Dong, Senior Member, IEEE, Wenming Lin, and Di Zhu, Member, IEEE
}

\begin{abstract}
The first rotating fan-beam scatterometer (RFSCAT) will be launched onboard the Chinese-French Oceanic Satellite (CFOSAT) in 2018. It provides a set of radar cross-section $\left(\sigma^{0}\right)$ measurements at different azimuth/incidence angles over a wind vector cell (WVC), in order to determine the near-surface wind field using the backscatter model, i.e., the so-called geophysical model function (GMF). The accuracy of the retrieved wind vector is a sensitive function of the radiometric accuracy of the $\sigma^{0}$ measurements. Therefore, in-flight calibration, including the loop-back (internal) calibration and the external calibration performed with natural extended-area targets, is studied in this paper. Several homogeneous areas over land are first analyzed to check the stability and azimuthal dependence of the $\sigma^{0}$ over these areas. A new calibration mask of the homogeneous land areas is generated and will be used by RFSCAT calibration. Then a simple method of external calibration is proposed to eliminate the azimuthal-dependent $\sigma^{0}$ errors induced by the insertion loss of the rotating joint, which can be applied to both the rotating pencil-beam scatterometers and the coming RFSCAT. The "observed" $\sigma^{0}$ of RFSCAT is simulated using the SeasatA scatterometer (SASS) measurements and the "perturbed" azimuthal-dependent $\sigma^{0}$ errors. The latter is then tracked by the proposed external calibration. The results show that the accuracy of gain corrections is up to $0.2 \mathrm{~dB}$, ensuring consistency between different azimuthal measurements.
\end{abstract}

Index Terms-External calibration, homogeneous areas, inflight calibration, internal calibration, rotating fan-beam scatterometer (RFSCAT).

\section{INTRODUCTION}

$\mathbf{S}$ PACEBORNE scatterometers are known to provide frequent coverage and wide-area measurements of ocean surface wind vectors. The latter, as an essential ocean dynamic parameter, improves the numerical weather predicting (NWP) models [1], [2]. The direct acquirement of scatterometer is the backscattered energy from the surface, which is then transferred into the normalized radar cross section $\left(\sigma^{0}\right)$ using the radar equation. After that, the equivalent neutral wind is

Manuscript received January 13, 2014; revised April 09, 2014; accepted June 20, 2014. Date of publication July 16, 2014; date of current version February 09, 2015. This work was supported by the National Natural Science Foundation of China under Grant 41006109.

J. Zhu is with the Key Laboratory of Microwave Remote Sensing, National Space Science Center, Chinese Academy of Sciences, Beijing 100190, China, and also with the University of Chinese Academy of Sciences, Beijing 100049, China (e-mail: jintai.hust@gmail.com).

X. Dong and D. Zhu are with the Key Laboratory of Microwave Remote Sensing, National Space Science Center, Chinese Academy of Sciences, Beijing 100190, China (e-mail: dongxiaolong@mirslab.cn).

W. Lin is with the Institute of Marine Sciences (ICM-CSIC), Barcelona 08003, Spain.

Color versions of one or more of the figures in this paper are available online at http://ieeexplore.ieee.org.

Digital Object Identifier 10.1109/JSTARS.2014.2333241 retrieved from a set of $\sigma^{0}$ measurements from diverse azimuth angles, according to the geophysical model function (GMF). Accuracy of the retrieved winds depends on the accuracy of $\sigma^{0}$ measurements. Therefore, precise calibration of those quantities used to calculate $\sigma^{0}$ through the radar equation is required, which mainly includes calibration of the antenna gain pattern, the atmospheric losses during the propagation of electromagnetic waves, the transmitted power, and the internal attenuation factors associated to the radar transmitting and receiving paths.

The prelaunch calibration campaigns alone are not able to achieve the desired accuracy of wind retrieval, i.e., $\pm 2 \mathrm{~m} / \mathrm{s}$ or $10 \%$ for wind speed retrieval and $\pm 20^{\circ}$ for wind direction retrieval. Thus, the postlaunch calibrations are used in all the operational scatterometer missions. Primarily, an internal calibration loop is designed to calibrate the transmitted power and the internal attenuation factors. It is implemented by coupling the transmitted power from the traveling wave tube amplifier (TWTA) into the receiver with predetermined insertion and attenuation losses. Besides, three key approaches of the postlaunch calibration (mainly for the antenna gain) have been drawn for the past and ongoing scatterometer missions, i.e., homogenous extended-area targets, active and/or passive ground calibration stations, and numerical ocean calibration [3]-[7]. Regarding the first approach, potential calibration sites must exhibit azimuthal-independent and spatially/temporally stable radar response over a large area [8]. Kennet and Li analyzed Seasat-A scatterometer (SASS) $\sigma^{0}$ data, which showed that Amazon and Congo rainforest, Sahara desert, Antarctica, and Greenland ice sheets are remarkably homogenous over a large area [8]. In this research, the backscatter characteristics of these areas are reviewed using QuikSCAT scatterometer image reconstruction (SIR) data provided by the Scatterometer Climate Record Pathfider (SCP) and Level-1B (L1B) $\sigma^{0}$ measurements from the HY-2A scatterometer. The second and third approaches have been well described by Yoho and Long [9], Wilson et al. [6], and Stoffelen [7], which are not presented in this study.

Rotating fan-beam scatterometer (RFSCAT) is a newly proposed radar scatterometer system for ocean surface wind vector measurement. The antenna beam sweeps the earth surface in a conical observing style like SeaWinds, but covers a much wider range of incidence angles. The first RFSCAT will be launched onboard the Chinese-French Oceanic Satellite (CFOSAT) in 2018. Comparing to the multiple fixed fan-beam scatterometers and the rotating pencil-beam scatterometers, RFSCAT has a more flexible observing geometry, i.e., the 
number of acquisitions and the observing azimuth angles vary with the cross-track location of the wind vector cell (WVC). This shows potential abilities in improving the accuracy of retrieved wind vectors [10], [11]. Furthermore, the multiple observations with different incidence angles over a certain WVC open a way to study the rain impact on the backscatter of radar scatterometer. The particular characteristics of RFSCAT geometry call for specific requirements in the calibration campaigns, beside the traditional calibration approaches used by the multiple fixed fan-beam scatterometer and the rotating pencil-beam scatterometer [10]. For example, the in-orbit loading force distribution of the rotary joint is definitely different from the on-ground measurements, thus the in-orbit insertion loss of the rotary joint may have different behaviors with the prelaunch measurement, and need to be calibrated during the $\sigma^{0}$ calculation.

This paper presents the preliminary study of the calibration for the coming RFSCAT onboard CFOSAT. In Section II, the RFSCAT instrument and the error sources of $\sigma^{0}$ measurements are introduced. Different types of data sources used in this paper are also presented in this section. The calibration methods of RFSCAT, including the internal calibration and the external calibration using extended-area targets, are described in Section III. The internal calibration of RFSCAT aims at calibrating the uncertainties introduced by the transmitted power and the internal attenuation factors. The proposed external calibration method is not designed for absolute calibration but is a relative calibration method designed to ensure consistency between measurements from diverse azimuth angles. Then the results are presented in Section IV. The potential calibration sites are first overviewed, and the homogenous properties are studied to find suitable land targets for the RFSCAT external calibration. The new calibration masks of homogenous sites are generated. Then the calibration results of HY-2A satellite scatterometer, which can be treated as a special case of rotating scatterometer, are analyzed using the proposed external calibration method in Section III. Finally, the experimental results of RFSCAT are presented based on a simulation approach. The summary is given in Section V.

\section{BACKGROUND}

\section{A. RFSCAT/CFOSAT}

The scatterometer onboard CFOSAT, which is a polarorbit satellite flying at an altitude of $519 \mathrm{~km}$ and an inclination of $97^{\circ}$, will be the first spaceborne RFSCAT for ocean surface wind vector measurement. It is a real aperture radar operating at $\mathrm{Ku}$-band $(13.256 \mathrm{GHz})$ frequency using both vertically and horizontally polarized antenna beams (i.e., $\mathrm{VV}$ and $\mathrm{HH}$ beams). It transmits long pulses with linear frequency modulation, and processes the received echoes by a dechirping technique. The frequency of the dechirped signal can be mapped into slant range through spectral analysis. Such procedure is in effect of pulse compression, which is achieved by Fourier transformation and squared modulus detection. The range resolution of each fast Fourier transformation (FFT) bin is about several hundred meters. The azimuth resolution of the measurements is determined by the antenna beamwidth

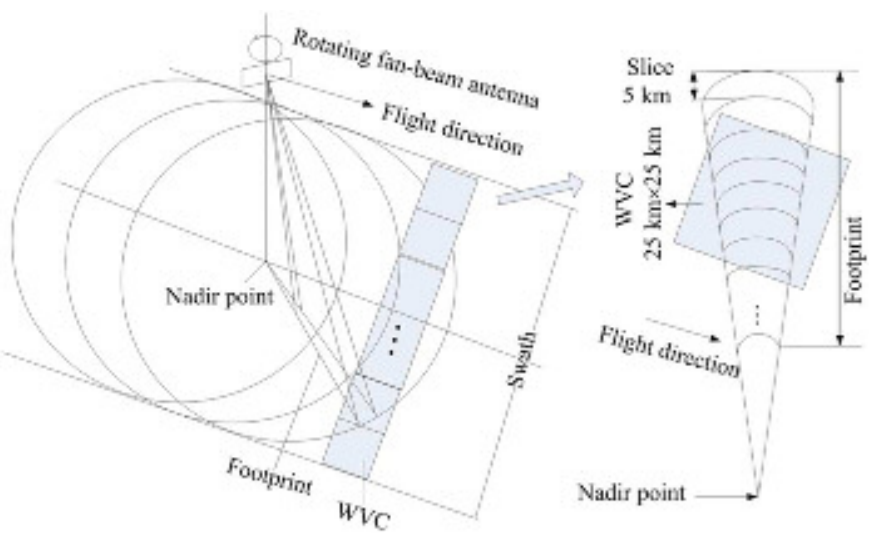

Fig. 1. Observe geometry of RFSCAT.

TABLE I

PRINCIPAL PARAMETERS OF RFSCAT

\begin{tabular}{ll}
\hline Specification & Valuc \\
\hline Frequency & $13.256 \mathrm{GHz}$ \\
\hline Bandwidth & $0.5 \mathrm{MHz}$ \\
\hline Polarizations & $\mathrm{HH}$ and VV \\
\hline Swath width & $>1000 \mathrm{~km}$ \\
\hline Orbit height & $519 \mathrm{~km}$ \\
\hline Inclination & $97^{\circ}$ \\
\hline Incidence angles & $26^{\circ} \sim 51^{\circ}$ \\
\hline Wind vector cell (WVC) resolution & $25 \mathrm{~km} / 50 \mathrm{~km}$ \\
\hline Rotation rate & $3.5 \mathrm{rpm}$ \\
\hline Pulse width & $1.35 \mathrm{~ms}$ \\
\hline Pulse repetition frequency (PRF) & $150 \mathrm{~Hz}$ \\
\hline Noise figure and losses within instrument & $5 \mathrm{~dB}$ \\
\hline
\end{tabular}

in the azimuth direction. Signals are further averaged into $5 \mathrm{~km}$ spaced slices along-elevation before being transmitted to ground, together with the data of noise measurements and internal calibration. The above-mentioned processing is adopted to reduce the data transmission in the link between satellite and ground segment. Fig. 1 illustrates the observation geometry of RFSCAT. The center of each fan beam has a nominal off-nadir angle of $40^{\circ}$. The incidence angle of RFSCAT beams has a range $26^{\circ}-51^{\circ}$. The highest incidence angle of $51^{\circ}$ and a mean platform altitude of $519 \mathrm{~km}$ ensure that the swath is about $1000 \mathrm{~km}$. Table I shows the main system parameters of RFSCAT.

\section{B. Error Sources of $\sigma^{0}$ Measurements}

The ground processing segment spatially averages the highresolution slices across- and along-track directions to obtain a set of $\sigma^{0}$ values for each WVC with desired resolution of 50 or $25 \mathrm{~km}$. The weighting function used to carry out the spatial filtering is a two-dimensional rectangular window or hamming window centered at every WVC position. Usually, the variability of each mean $\sigma^{0}$ measurements is described by the relative deviation $K_{p}$, which is an estimate of the normalized standard deviation of the measured $\sigma^{0}$ values [3], [12]. Low $K_{p}$ value corresponds to high accurate backscatter estimate, and vice versa. The $K_{p}$ used in the wind retrieval is normally decomposed into three independent variability contributions, i.e., communication error (radiometric noise, $\left.K_{p c}\right)$, retrieval or calibration error $\left(K_{p r}\right)$, and modeling error 


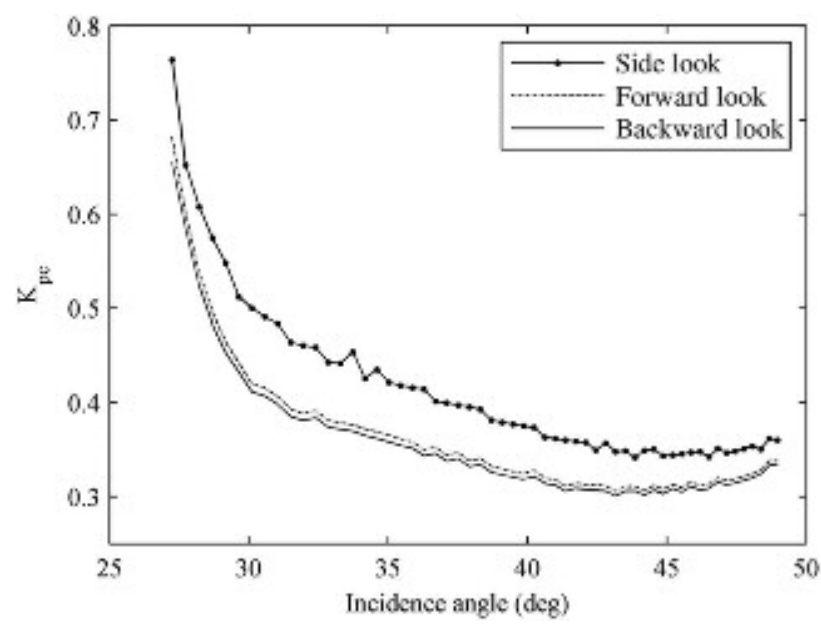

Fig. 2. RFSCAT $K_{\mathrm{pc}}$, as a function of incidence angles for three typical azimuth angles, is estimated from the backscatter measurements over Amazon rainforest.

$\left(K_{p m}\right)$. This is expressed as [3]

$$
K_{p}=\sqrt{K_{p c}^{2}+K_{p r}^{2}+K_{p m}^{2}}
$$

$K_{p c}$ is a function of the measurement signal-to-noise ratio (SNR) and the number of independent samples within a certain WVC [13]. It is an inherent factor of scatterometer measurements for a given set of observing geometry and scatterometer parameters. A comprehensive study of the relationship between the RFSCAT instrument parameters and the $K_{p c}$ value has been performed in [11] and [13], where $K_{p c}$ is simply estimated by the normalized standard deviation of the backscattered signal power for a certain slice. Since the Doppler frequency changes as the antenna rotates, the number of FFT bins and the slice band used to calculate $K_{p c}$ vary with the observing azimuth angles, and in turn, result in an azimuthal-dependent communication error. Fig. 2 illustrates the $K_{p c}$ as a function of incidence angles for three different azimuth angles, which estimated from the slice measurements over Amazon rainforest.

The calibration error $K_{p r}$ is due to the errors in the parameters of radar equation that are used to calculate $\sigma^{0}$ [3]. All the instrument parameters in the radar equation (i.e., antenna gain pattern, the gain of receiver, system losses, and transmitted power) impact $\sigma^{0}$, so they need to be calibrated through prelaunch or postlaunch campaigns and to be compensated in the data processing. The antenna gain pattern is obtained during the prelaunch laboratory experiment and will be further calibrated using the extended land targets through the whole CFOSAT mission. The receiver gain and the transmitted power are time-varying and temperature-dependent parameters. They are also tested in the prelaunch experiment and are compensated through the scatterometer internal calibration loop. The insertion loss of rotary joint, which introduces azimuthaldependent fluctuations, is measured at prelaunch stage and will be further monitored and calibrated using both the calibration ground station and the natural extended-area targets. The transmitting/receiving channels and onboard processing may drift during the life time of the instrument, and they will be calibrated by combination of onboard internal calibration and calibration ground stations [10]. In addition, the satellite attitude error is another factor impacting on the calculation of $\sigma^{0}$. The objective of calibration is to minimize all these effects and thereby to reduce the $K_{p r}$ error.

$K_{p m}$ associates to the spatial and temporal variability inherent to the target. For instance, over the ocean it is due to the factors not accounted in the GMF, mainly the subcell wind variability in the absence of rain [14]. For the calibration campaign over the land, $K_{p m}$ can be reduced to a proper value by carefully choosing the extended targets that exhibit high $\sigma^{0}$ stability.

\section{Data}

HY-2A scatterometer is onboard the first ocean dynamic monitoring satellite of China, HY-2A satellite, together with other three microwave payloads, which are the radar altimeter, the scanning microwave radiometer, and the three-frequency microwave radiometer [15]. The backscatter measurements of HY-2A scatterometer and SASS, together with the QuikSCAT SIR data, are used in this study. SIR data are images generated via the SIR algorithm available from the SCP [16]. Using the SIR algorithm, different orbits for QuikSCAT during a 4-day span are combined to create separate ascending and descending data images for different regions. The SIR data images measured by QuikSCAT in 2003 and 2009 have been used in this paper to detect the temporally stable signatures over different calibration regions.

After coarsely identifying the homogeneous regions using QuikSCAT SIR data, a finer selection of land targets for RFSCAT/CFOSAT calibration is applied by analyzing the targets' responses of the recent $\mathrm{HY}-2 \mathrm{~A}$ scatterometer observation during March, 2012. The L1B data of HY-2A scatterometer is adopted in the study.

Due to the absence of the ongoing Ku-band fan-beam scatterometer, the backscatter measurements of RFSCAT are simulated using the actual SASS measurements over Amazon rainforest during 1978 .

\section{RFSCAT CALIBRATION METHOD}

\section{A. Internal Calibration}

Internal calibration is one of the nominal operation modes of any spaceborne scatterometer. It is performed onboard to monitor the combined variations of the transmitted power and the receiver gain. The implementation of internal calibration is shown in Fig. 3. Besides the regular transmission of microwave pulses (indicated by the red line), for some pulses, the transmitted power is coupled into the receiver through a set of couplers with attenuation coefficient of $-110 \mathrm{~dB}$ (indicated by the green line). The coupled signal is then downconverted and processed using the same circuit as what is used to process the scatterometer echoes (represented by the blue line). Downlinked internal calibration data are used to correct variations mentioned above during the ground processing, which is achieved by a ratio method [17].

The clutter by coupling outside the calibration loop is tested in the laboratory under different temperature conditions. Its 


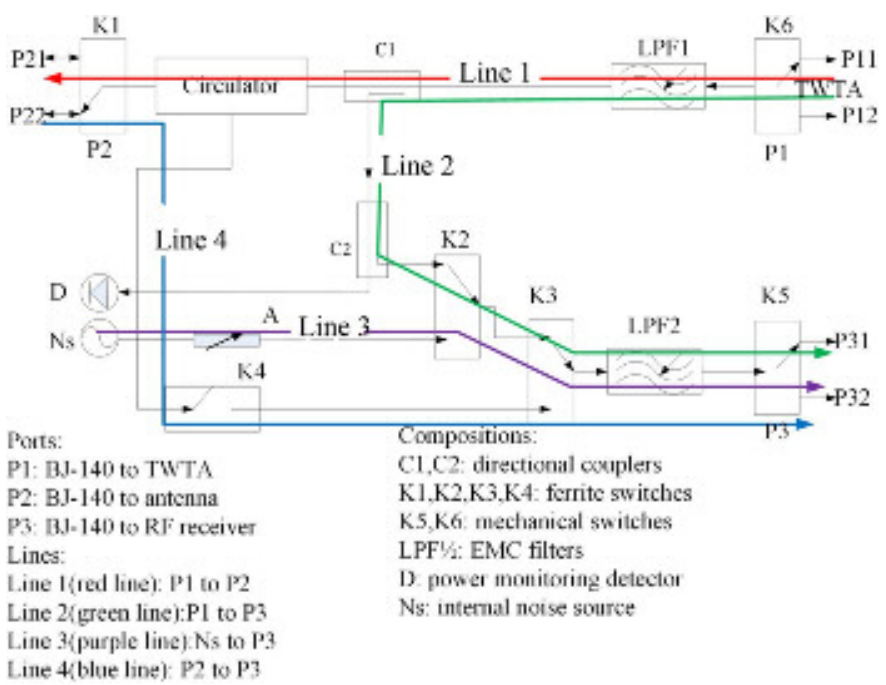

Fig. 3. Signal flow of internal calibration loop. The red line (Line 1) represents the transmission channel of pulses that are transmitted by TWTA. The green line (Line 2) indicates the signal loop of internal calibration, and the signal is coupled from the transmitted power by couplers. The purple line (Line 3) is the noise measurement channel of the internal calibration and the noise is generated by an internal noise source. The blue (Line 4) is the echo channel of the signal that is backscattered from the targets.

measured value is less than $-130 \mathrm{~dB}$. Thus the clutter of calibration signal received through the blue line in Fig. 3 is more than $20 \mathrm{~dB}$ lower than the power coupled from the internal calibration loop, which leads to an uncertainty of about $0.05 \mathrm{~dB}$. The in-band fluctuations of the transmitting/receiving channels are measured in the prelaunch thermal-vacuum test under different temperature conditions. The measurement precision for passive part of the transmitting/receiving channels is less than $0.1 \mathrm{~dB}$ after thermal compensation. There is a programmable gain controller (PGC) inside the receiver, the absolute insertion loss of which may vary with the temperature inside the receiver. Thus, a lookup table of the PGC gain is established during the prelaunch test campaign. The realtime PGC gain is obtained by interpolating the table to the value acquired by the temperature telemetry. After thermal compensation, the PGC inside the receiver has an accuracy of $0.1 \mathrm{~dB}$. In summary, the overall internal calibration error is less than $0.15 \mathrm{~dB}[17]$.

\section{B. External Calibration}

External calibration is adopted to eliminate the inconsistencies among the $\sigma^{0}$ measurements acquired from different azimuth angles and over the whole measurement incidence angle range. The former corresponds to the calibration of insert loss of the rotary joint, as well as the azimuthal-dependent biases introduced by the satellite attitude uncertainty, and the latter associates with the calibration of antenna gain pattern. To simplify the calibration procedure, a total error approach is used to model the errors mentioned above. The measured parameter is assumed to be $\sigma_{\text {meas }}^{0}$ with all error sources supposed to be additive in log space, i.e., to be multiplicative in natural space. The additive errors in natural space are ignored, because they affect the returned power (indicated by the blue line in Fig. 3) and the noise estimation (indicated by the purple line in Fig. 3) simultaneously and is eliminated when calculating $\sigma^{0}$. Therefore, the measurement of $\sigma^{0}$ is described as following:

$$
\begin{aligned}
\log _{10}\left(\sigma_{\text {meas }}^{0}\right)= & \log _{10}\left(\sigma_{t}^{0}\right)+\log _{10}\left(\sigma_{b}^{0}\right) \\
& +\log _{10}\left(\sigma_{r}^{0}\right)+\log _{10}\left(\sigma_{\text {noise }}^{0}\right)
\end{aligned}
$$

where $\sigma_{t}^{0}$ is the true $\sigma^{0}$ value of the calibration targets, $\sigma_{b}^{0}$ is the total end-to-end system gain bias (mainly due to the antenna gain bias), which equally affects the azimuthal backscattering measurements of RFSCAT. $\sigma_{r}^{0}$ is the relative bias for the $\sigma^{0}$ observed at a particular azimuth angle (mainly due to the insert loss of rotary joint and the antenna pointing errors) and varies periodically as the antenna rotates. $\sigma^{0}$ noise is a zero-mean term, which is used to model the random uncertainties caused by thermal noise. The effect of $\sigma^{0}$ noise is damped by averaging the measured backscattering over sufficient samples.

In general, the bias $\sigma_{b}^{0}$ impacts the retrieval of wind speed, and the relative bias $\sigma_{r}^{0}$ affects the retrieval of wind direction. A radiometric error with a value of $0.2 \mathrm{~dB}$ in the bias $\sigma_{b}^{0}$ results in about $0.2 \mathrm{~m} / \mathrm{s}$ wind speed effects, while the relative bias $\sigma_{r}^{0}$ of the same amount may lead to notable wind direction effects [7], [18]. In case that extended land targets are used for the external calibration, it is difficult to know the exact value of $\sigma_{t}^{0}$, so there is no accurate way to determine $\sigma_{b}^{0}$. In this paper, we concentrate on determining the relative bias $\sigma_{r}^{0}$. The contributions of $\sigma_{t}^{0}$ and $\sigma_{b}^{0}$ are treated as effective $\sigma^{0}$, i.e., $\sigma_{\text {eff }}^{0}$, in total. Thus, the measured $\sigma^{0}$ becomes

$$
\log _{10}\left(\sigma_{\text {meas }}^{0}\right)=\log _{10}\left(\sigma_{\text {eff }}^{0}(\theta)\right)+\log _{10}\left(\sigma_{r}^{0}(\phi, \theta)\right)
$$

where $\theta$ and $\phi$ are incidence angle and azimuth angle, respectively.

Following the methodology developed by Long and Skouson [3], [19], a fourth-order polynomial is adopted to model the incidence angle response, which is consistent with the fact that $\sigma^{0}$ of the calibration target is a smooth function of incidence angles. Meanwhile, in order to characterize the azimuthal-dependent $\sigma_{r}^{0}$ value, the azimuth angles of the antenna beam are separated into 24 bins (with $15^{\circ}$ bin width), which are sufficient for correcting the expected relative bias. They are numbered from 1 to 24 , i.e., 1 corresponds to azimuth angle $\left[0^{\circ} 15^{\circ}\right), 2$ corresponds to azimuth angle $\left[15^{\circ} 30^{\circ}\right)$, and so on. Thus, the measured $\sigma^{0}$ over the target is modeled as

$$
\begin{gathered}
10 \log _{10}\left(\sigma_{\text {meas }}^{0}\left(k, \theta_{n}\right)\right)=P_{\text {meas }}(0, k)+P_{\text {meas }}(1, k) \theta_{n} \\
+P_{\text {meas }}(2, k) \theta_{n}^{2}+P_{\text {meas }}(3, k) \theta_{n}^{3}+P_{\text {meas }}(4, k) \theta_{n}^{4}
\end{gathered}
$$

where $k=1, \ldots, 24 . P_{\text {meas }}$ are the polynomial coefficients associated with $\sigma_{\text {meas }}^{0}$, and $\theta_{n}$ is the incidence angle of the $n$th slice. Each term of $P_{\text {meas }}$ is decomposed into the sum of the polynomial coefficients associated with $\sigma_{e f f}^{0}$ (denoted as $P_{\text {eff }}$ ) and the one associated with $\sigma_{r}^{0}$ (denoted as $P_{r}$ ). Thus the regression model can be expressed as

$$
P_{\text {meas }}(i, k)=P_{\text {eff }}(i, k)+P_{r}(i, k), \quad i=0, \ldots, 4 .
$$


(a1)
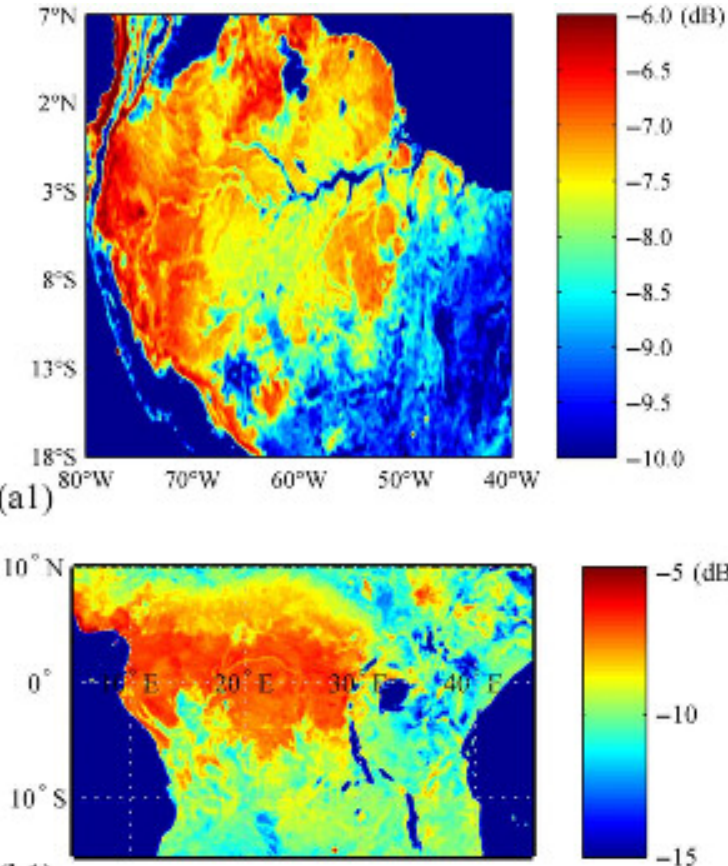

(b1)

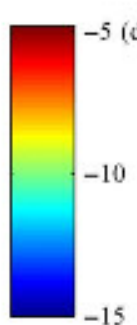

15

(b2)
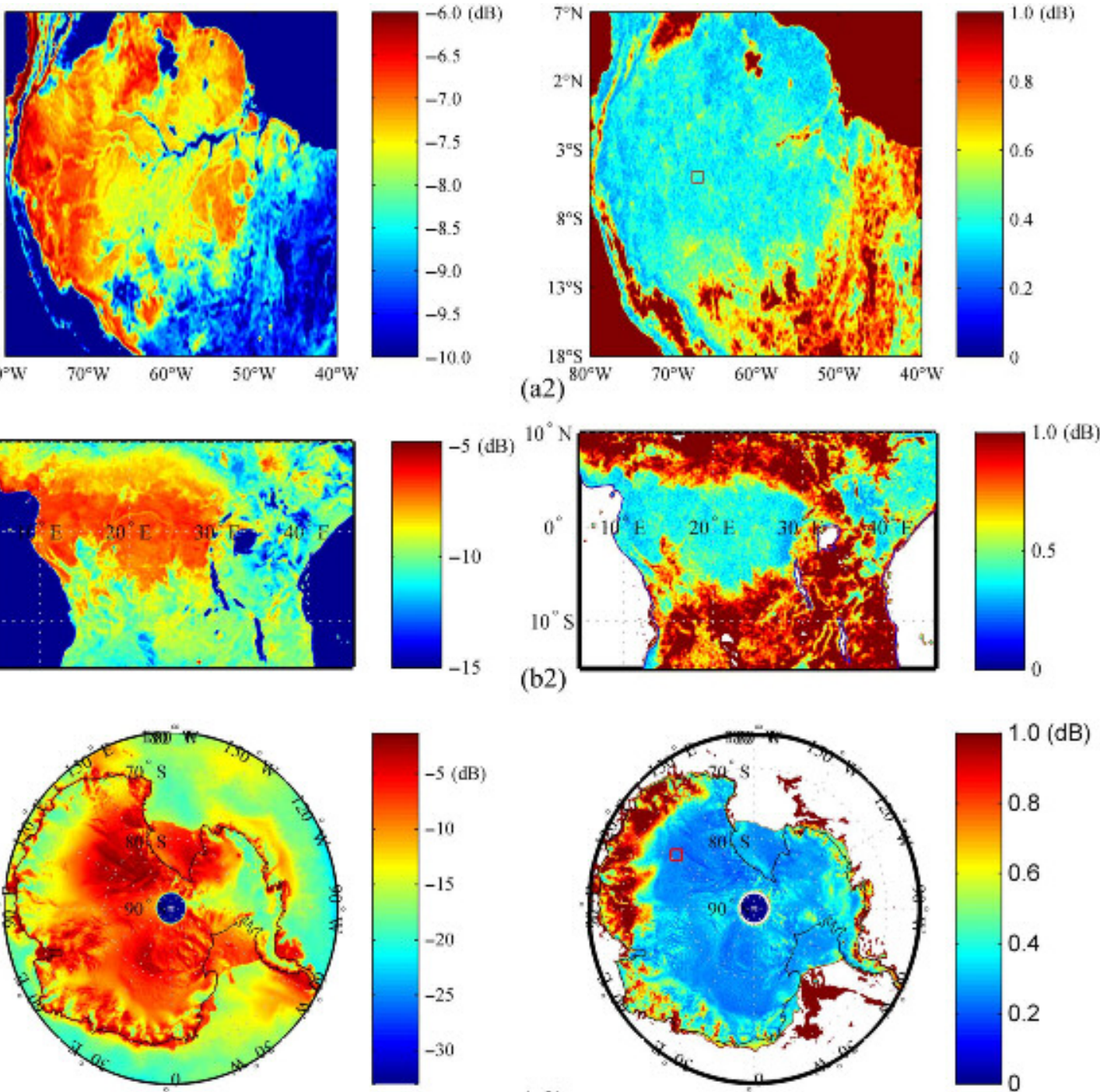

(c1)
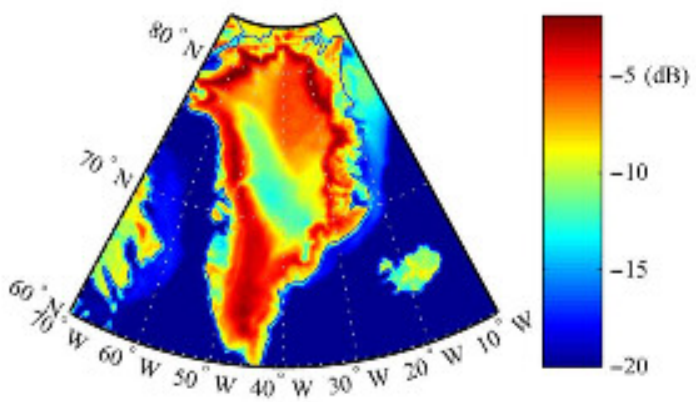

(d1)

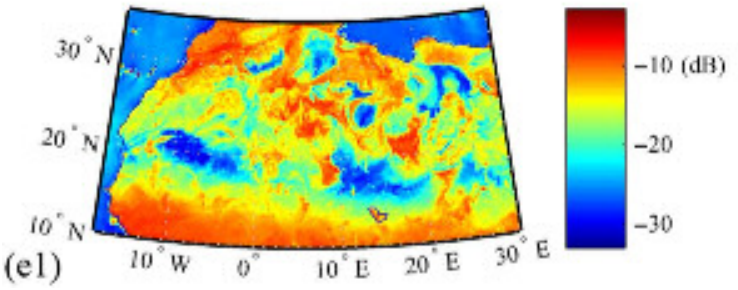

(c2)
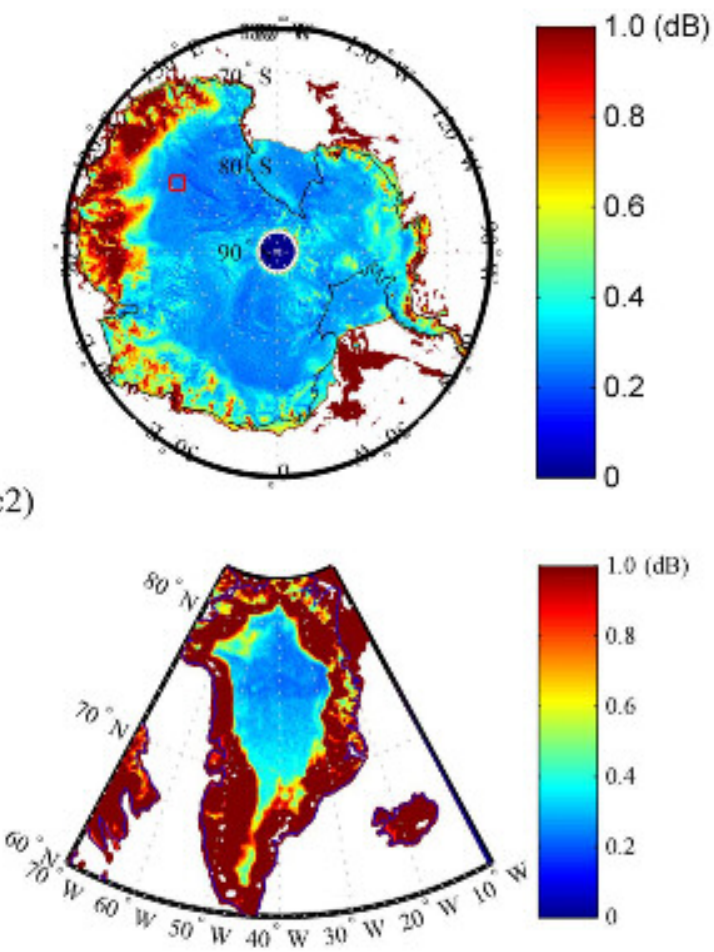

(d2)

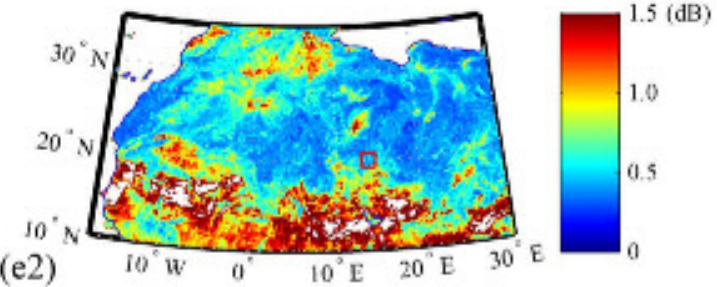

Fig. 4. Average and the standard deviation of $\sigma^{0}$ for HH-polarized QuikSCAT in 2009 over (a) Amazon, (b) Congo, (c) Antarctic, (d) Greenland, and (e) Sahara. The left figures are average $\sigma^{0}$ and the right figures are the standard deviation, respectively. 
TABLE II

Statistics of the Monthly Averaged Backscatter ACQuired by HY-2A SCATTERometer OVER the CANDIDATE Calibration AREAS

\begin{tabular}{lccccc}
\hline Region & Pass & \multicolumn{2}{c}{ VV polarization } & \multicolumn{2}{c}{ HH polarization } \\
\cline { 3 - 6 } & & $\begin{array}{c}\text { Average } \\
\text { (dB) }\end{array}$ & Std Dev & $\begin{array}{c}\text { Average } \\
\text { (dB) }\end{array}$ & Std Dev \\
\hline \multirow{2}{*}{ Amazon } & Asc & -8.35 & 0.43 & -7.67 & 0.41 \\
\cline { 2 - 6 } & Des & -8.14 & 0.40 & -7.29 & 0.42 \\
\hline Congo & Asc & -8.34 & 0.43 & -7.94 & 0.43 \\
& Des & -7.94 & 0.39 & -7.38 & 0.41 \\
\hline \multirow{2}{*}{ Antarctic } & Asc & -5.55 & 0.40 & -5.65 & 0.40 \\
& Des & -5.47 & 0.39 & -5.66 & 0.41 \\
\hline Greenland & Asc & -5.20 & 0.39 & -5.83 & 0.36 \\
& Des & -5.14 & 0.30 & -5.64 & 0.36 \\
\hline Sabural & Asc & -22.48 & 0.84 & -21.57 & 0.85 \\
\hline & Des & -22.53 & 0.87 & -21.58 & 0.90 \\
\hline
\end{tabular}

The HY-2A Data was acquired in March, 2012.

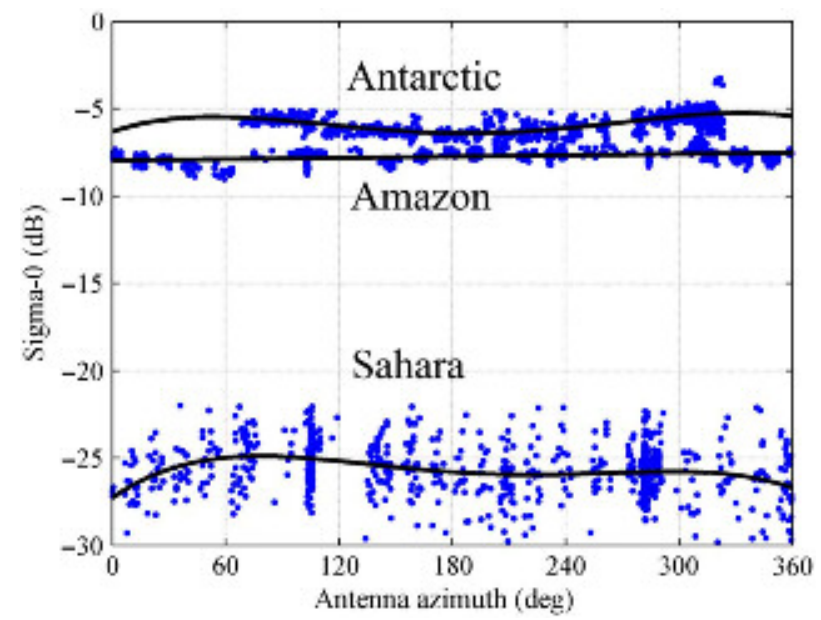

Fig. 5. Variation in $\sigma^{0}$ with azimuth angles in ascending pass for VVpolarized of HY-2A scatterometer in March, 2012.

The relative bias $\sigma_{r}^{0}$ and its polynomial coefficients $P_{r}$ can only be properly defined with respect to a reference beam $\left(b_{r e f}\right)$, whose $\sigma^{0}$ measurements are assumed to be correct, i.e., $\sigma_{r}^{0}\left(\theta_{n}, b_{r e f}\right)=0$ in (3). Thus, $P_{r}\left(i, b_{r e f}\right)$ equals to zeros for $i=0, \ldots, 4$. The mean value of $\sigma_{\text {meas }}^{0}$ that measured over all azimuth angles is used as the reference beam in this paper. Subtracting (5) from itself with $k=b_{\text {ref }}$ for all $k$ gives

$$
\begin{gathered}
P_{\text {meas }}(i, k)-P_{\text {meas }}\left(i, b_{\text {ref }}\right)=P_{r}(i, k) \\
\text { or } \\
\Delta P_{\text {meas }}(i, k)=P_{r}(i, k) .
\end{gathered}
$$

Then the regression coefficients $P_{r}(i, k)$ can be retrieved by generalized inverse solution and the estimations of the relative biases $\hat{\sigma}_{r}^{0}$ for the $\sigma^{0}$ overall azimuth angles are depicted as follows:

$$
10 \log _{10}\left(\hat{\sigma}_{r}^{0}\left(k, \theta_{n}\right)\right)=\sum_{i=0}^{4} P_{r}(i, k) \bullet \theta_{n}^{i} .
$$

Estimations of the relative biases derived from (7) are subtracted from raw $\sigma^{0}$ measurements in $\log$ space (i.e., $\left.10 \log 10\left(\sigma^{0}\right)\right)$ to achieve backscatter consistency among different antenna azimuth bins. This correction method can eliminate the azimuthal-dependent $\sigma^{0}$ bias and verify the RFSCAT

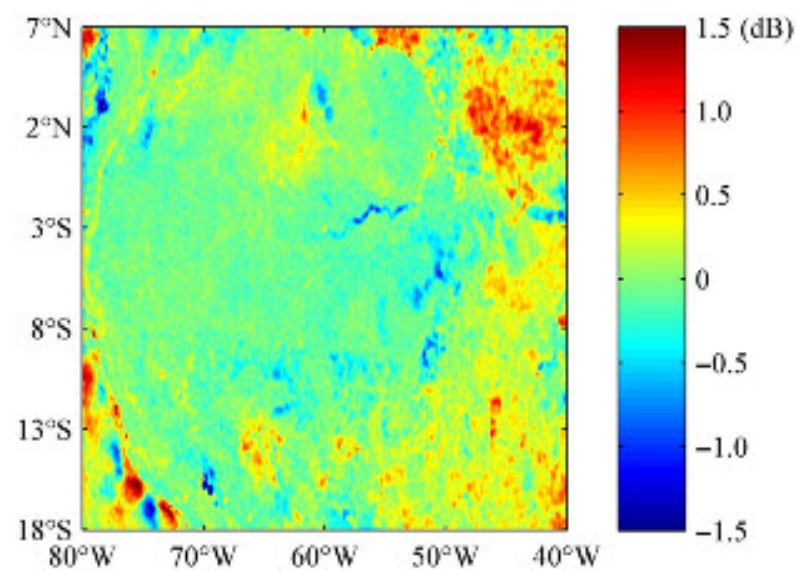

Fig. 6. Illustration of the $\sigma^{0}$ difference between 2003 and 2009 over the Amazon rainforest.

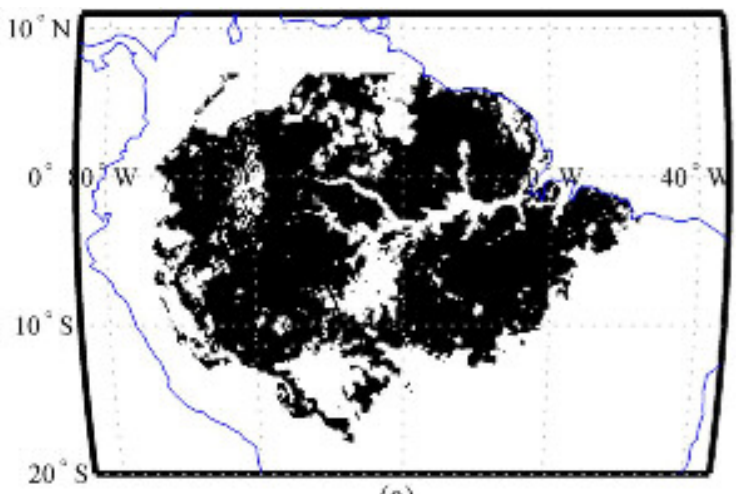

(a)

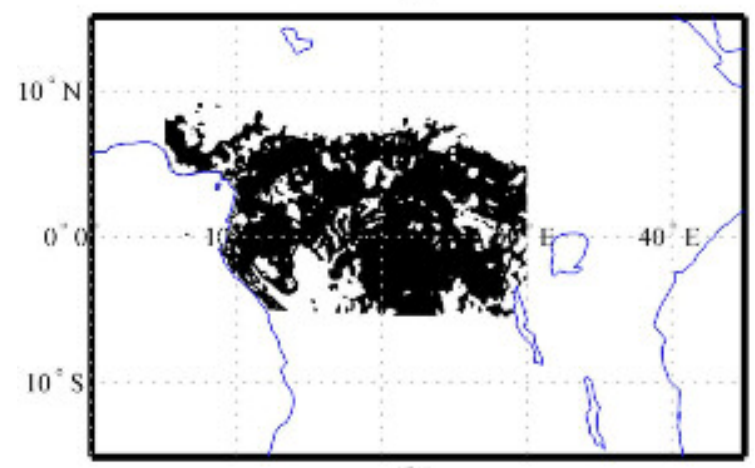

(b)

Fig. 7. Map masks (black areas) of (a) the Amazon rainforest and (b) the Congo rainforest, which correspond to a high spatial and temporal stability. The masks correspond to those pixels with a temporal standard deviation $<0.5 \mathrm{~dB}$.

antenna gain fluctuation induced by the azimuthal-dependent insertion loss of the rotating joint.

\section{Simulation And Results}

\section{A. Calibration Regions}

Areas for external calibration should be temporally and spatially stable, and exhibit isotropic radar response (i.e., azimuthal-independent). Ideally, the calibration targets' backscatter only depends on the incidence angle. According to the mentioned requirements, the Amazon tropical rainforest, the Congo rainforest, the Sahara desert and the internal 

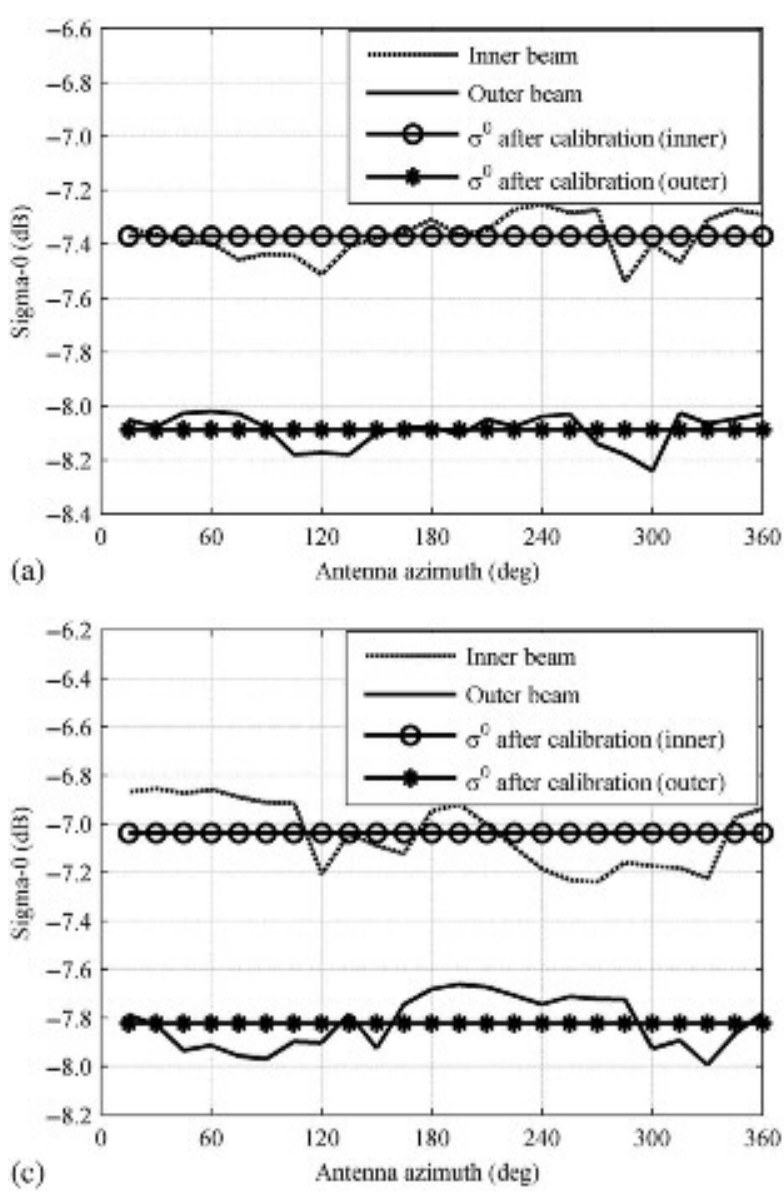

(c)

Fig. 8. HY-2A scatterometer beam azimuth balance over Amazon rainforest. (c) $\sigma^{0}$ of the descending pass. (d) Beam corrections for the descending pass.

Antarctic, and Greenland ice shells are good candidates of the scatterometer calibration campaign in order to achieve the consistencies between $\sigma^{0}$ measurements observed at different azimuth angles and to verify the in-orbit antenna gain pattern. The mean and the standard deviation of $\sigma^{0}$ of these candidate sites are illustrated in Fig. 4 using the QuikSCAT SIR data.

Table II presents the statistics of the backscatter measurements acquired by HY-2A scatterometer over the candidate calibration areas. The small standard deviations of $\sigma^{0}$ measured by HY-2A scatterometer over these regions are consistent with Fig. 4. The backscatter measurements over Antarctic and Greenland ice shells are larger than the other targets due to the higher reflectance of ice shells, and the standard deviation are much less due to the permanent feature of ice shells [20]. The standard deviation of $\sigma^{0}$ over Sahara desert is the largest over these calibration candidates. Fig. 5 shows the variation of $\sigma^{0}$ with azimuth angles over some selected areas with small standard deviations. The locations of those selected areas plotted in Fig. 5 are $\left(\left[4.5^{\circ} \mathrm{S}, 5.5^{\circ} \mathrm{S}\right]\right.$, $\left.\left[66.5^{\circ} \mathrm{W}, 67.5^{\circ} \mathrm{W}\right]\right),\left(\left[75.5^{\circ} \mathrm{S}, 76.5^{\circ} \mathrm{S}\right],\left[122.5^{\circ} \mathrm{E}, 123.5^{\circ} \mathrm{E}\right]\right)$, and $\left(\left[19.5^{\circ} \mathrm{N}, 20.5^{\circ} \mathrm{N}\right],\left[13.5^{\circ} \mathrm{E}, 14.5^{\circ} \mathrm{E}\right]\right)$, respectively, and they are marked in red square in Fig. 4(a2), (c2), and (e2). There is a large anisotropic behavior in the Antarctic, as shown in Fig. 5, due to strong wind over the ice shell [21]. The usage of Antarctic ice shell for calibration requires
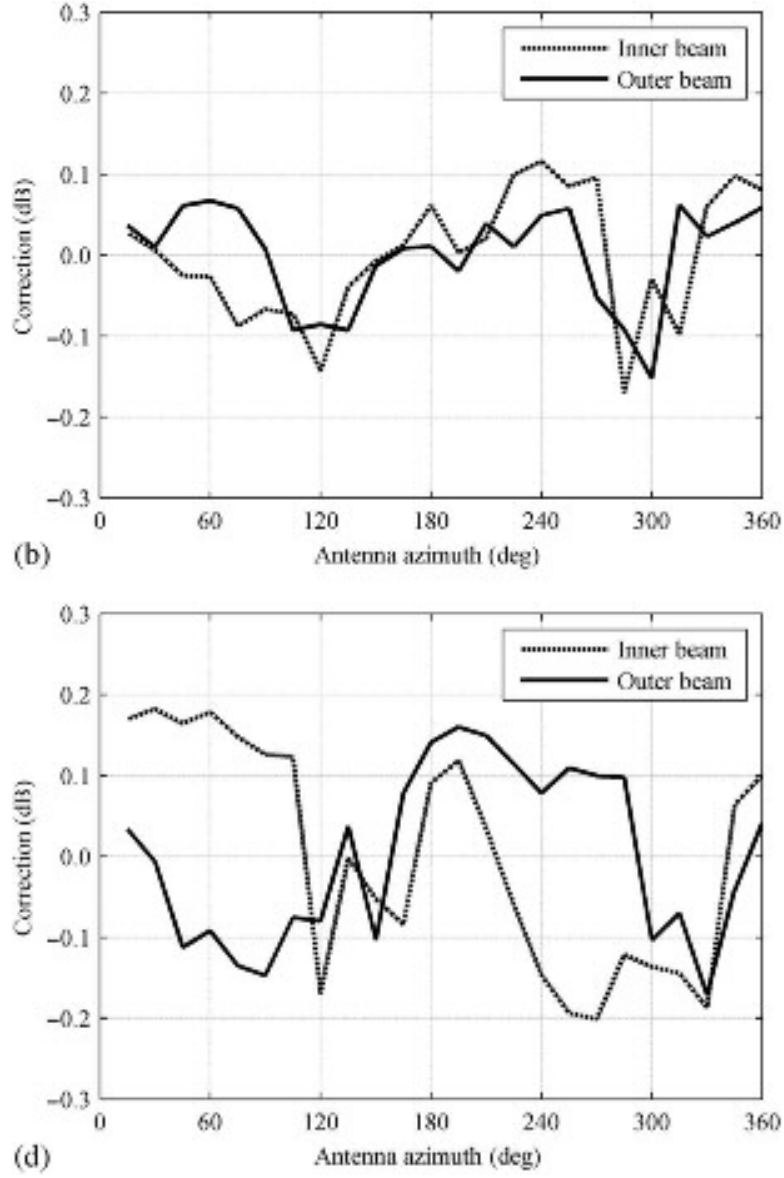

(a) $\sigma^{0}$ of the ascending pass. (b) Beam corrections for the ascending pass. more characterizations of anisotropic properties, which should be considered in the calibration; Regarding the Greenland, there is only a small area that can be used for calibration of pencil-beam scatterometer, but not suitable for fan-beam scatterometer with large footprint [17], [22].

Table II also shows that there is a difference of $\sim 0.5 \mathrm{~dB}$ between the ascending and descending passes over Amazon rainforest and Congo rainforest. However, this feature cannot be observed over Sahara desert and Greenland ice shell. This may be due to the diurnal moisture cycle caused by the different lighting conditions. The moisture transports from the canopy due to water vapor evaporation during daytime (descending pass) and dies out during the night (ascending pass), causing the diurnal variation of the $\sigma^{0}$ over these areas [3], [20], [23]. Due to deforestation of the rainforest, some parts of rainforest have been damaged and are not suitable for the calibration. The difference between QuikSCAT SIR data obtained in 2003 and 2009 are analyzed and the results are illustrated in Fig. 6, which shows that the radar response in most part of Amazon rainforest remain with high precision except the southeast part [5], [10], [23]. The new masks of Amazon rainforest and Congo rainforest, corresponding to spatially homogenous regions, are produced (Fig. 7) by employing the QuikSCAT SIR data measured during ascending pass in 2009 , and they can be used as references in the calibration of future scatterometers. 

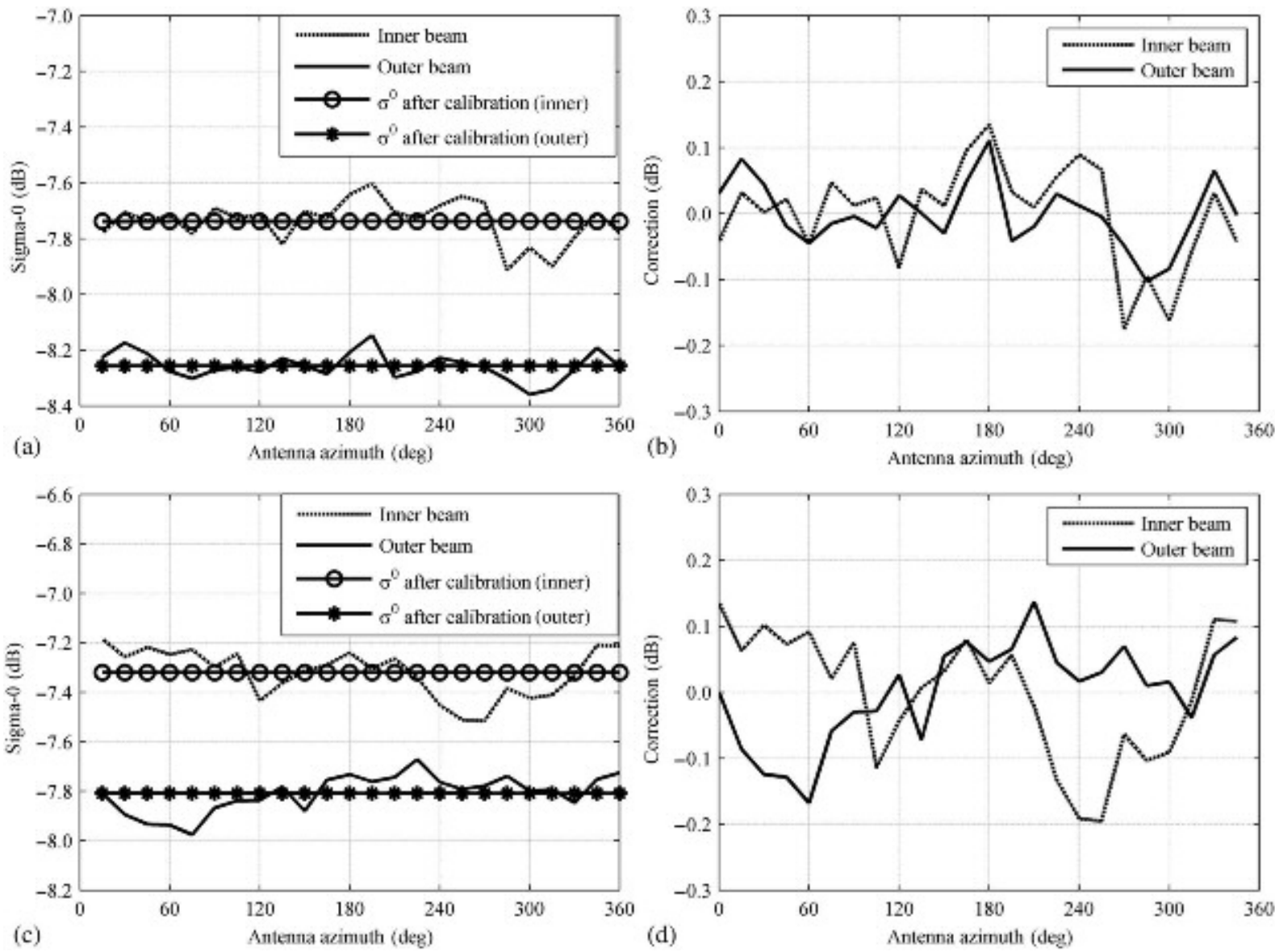

Fig. 9. HY-2A scatterometer beam azimuth balance over Congo rainforest. (a) $\sigma^{0}$ of the ascending pass. (b) Beam corrections for the ascending pass. (c) $\sigma^{0}$ of the descending pass. (d) Beam corrections for the descending pass.

Long-term temporal variations in $\sigma^{0}$ measurements over Amazon rainforest have been observed in the data from those past scatterometers [3], [4]. Increasing the amount of samples by extending the time window will introduce higher temporal variations into $\sigma^{0}$ measurements. A 2-week time window is adopted as a compromise between the amount of data and time stability.

\section{B. Rotating Pencil-Beam Scatterometer Results}

The HY-2A SCAT adopts two rotating pencil beams at two constant incidence angles $\left(41^{\circ}\right.$ for inner beam and $48^{\circ}$ for outer beam) to measure radar backscattering power [15]. Beam balance is achieved by comparing the backscatter data acquired as the antenna rotates with a reference azimuth interval. The mean value of all data measured over all azimuth angles $\left(0^{\circ}-360^{\circ}\right)$ are used as the reference. According to the analysis of HY-2A scatterometer data in March 1-March 16 (except March 7), 2012, the azimuthal uncertainties of raw $\sigma^{0}$ (before calibration) are within $\pm 0.3 \mathrm{~dB}$. The results are shown in Figs. 8 (Amazon rainforest) and 9 (Congo rainforest). The relative biases over different azimuth angles are due to the point errors of the rotating axis (equivalently, satellite attitude errors). The rotating axis of HY-2A SCAT is set up to be geocentric pointing, which would introduce azimuthal variations of the measurements during the rotation. Figs. 8(a) and (c) and 9(a) and (c) show the $\sigma^{0}$ azimuth responses of HY-2A scatterometer (dashed lines for inner beam and solid lines for outer beam), comparing to the calibration results over all azimuth angles (circle horizontal lines and star horizontal lines), whereas Figs. 8(b) and (d) and 9(b) and (d) show the correction values over all azimuth angles. The calibration masks used here are the Amazon rainforest mask and the Congo rainforest mask shown in Fig. 7.

The standard deviations of the $\sigma^{0}$ over Amazon rainforest or Congo rainforest after calibration are less than $0.1 \mathrm{~dB}$. By comparing Fig. 8(a) with Fig. 9(a), the diurnal change of $\sigma^{0}$ is observed. The difference $\sigma_{\text {des }}^{0}-\sigma_{\text {asc }}^{0} \approx 0.5 \mathrm{~dB}$ verifies the conclusion that is discussed in Section IV-A. The calibration values are different between the ascending pass and descending pass. These differences of calibration values are mainly introduced by: 1) the variations in the average orientation of the canopy leaves at different sun angles, 2) different instrument temperatures on the ascending and descending passes [3], and 3) the pointing errors of antenna. Since the rotating axis is in the geocentric pointing, there will be a variation of several tenths of a degree in the pointing angles of antenna during different satellite passes and different latitudes. Calibration values in the right figures of Fig. 8 have the similar tendencies with those in the right figures of Fig. 9, indicating that the azimuthal-dependent variations are likely to be caused by the instrument, i.e., the satellite pointing errors. 

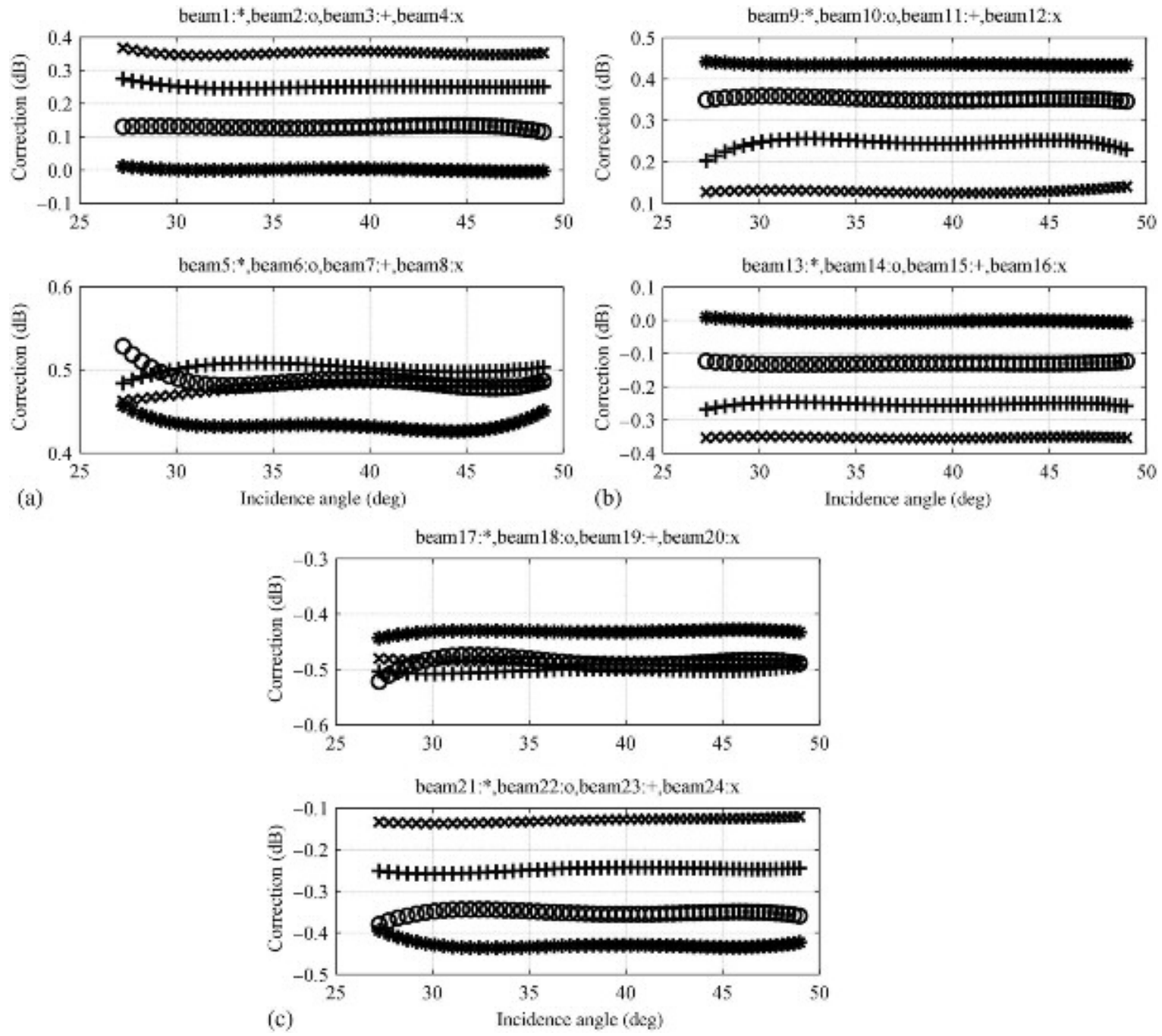

Fig. 10. Different beam corrections of 24 bins (azimuth bins). Beams $1-24$ correspond to azimuth angles $\left(0-360^{\circ}\right)$.

The minor differences between Figs. 8(b) and 9(b) [or Figs. $8(d)$ and $9(\mathrm{~d})]$ may be due to the fact that Amazon and Congo are different latitudes and the scene variations exist between Amazon and Congo.

\section{RFSCAT Results}

Due to the different loading force, the insertion loss of rotary joint is an azimuthal-dependent parameter, but it is considered to behave the same for a long time in space. Thus, natural extended-area targets will be used for the external calibration monthly throughout the whole CFOSAT mission.

Since there is no ongoing Ku-band fan-beam scatterometer, we use the fourth-order polynomial model coefficients of SASS's $\sigma^{0}(\theta)$ over Amazon rainforest to simulate the effective $\sigma_{\text {eff }}^{0}$, in which the coefficients are $P_{\text {eff }}(0)=-7.66$, $P_{\text {eff }}(1)=-1.079, P_{\text {eff }}(2)=0.121, P_{\text {eff }}(3)=-0.012$, and $P_{\text {eff }}(4)=0.079$, respectively [19]. Then Monte Carlo method is used to simulate the measured $\sigma^{0}$ which is perturbed by thermal noise, as supposed to be of Gaussian distribution with a zero-mean and a variance $K_{p c}$

$$
\sigma_{\text {meas }}^{0}=\sigma_{e f f}^{0}+\sigma_{r}^{0}+\sigma_{\text {eff }}^{0} \cdot N\left(0, K_{p c}\right)
$$

where $\sigma^{0}$ eff $\bullet N\left(0, K_{p c}\right)$ is the thermal noise. $\sigma_{r}^{0}$ is a periodic variation versus azimuth angle and is simulated using a sinusoidal function with the maximum magnitude of $0.5 \mathrm{~dB}$.

Using the calibration method described in Section III, corrections of the relative biases over different antenna beams (different azimuth bins) are given in Fig. 10. The standard deviation of the $\sigma^{0}$ after calibration is less than $0.06 \mathrm{~dB}$. Fig. 10 shows that there are some perturbations less than $0.05 \mathrm{~dB}$ in the corrections. The high perturbations mainly exhibit in the inner-swath and the out-swath of the footprint, which is primarily due to lower SNR in these parts (especially for the inner-swath part), than that near to the boresight observing direction. The larger values of $K_{p c}$ (Fig. 2) at the low incidence angles correspond well with the higher those perturbations.

As mentioned in Section IV-A, the selected calibration targets are remarkably homogenous. However, there are indeed small variations in the targets that would induce errors in the calibration. The residual variability of Amazon over the calibration mask is estimated to be $0.15 \mathrm{~dB}$ [3], suggesting that the accuracy of calibration using Amazon is no less than $0.15 \mathrm{~dB}$. Regarding that the temporally and spatially stable 
characteristics of Amazon have been proved by the past scatterometers, the calibration coefficients derived from Amazon are used for RFSCAT external calibration. Nevertheless, the other calibration targets are still useful to provide independent verifications.

\section{CONCLUSION}

In this paper, some considerations of the calibration for a Ku-band RFSCAT onboard CFOSAT are presented. The internal calibration of RFSCAT is briefly discussed in this paper. The overall internal calibration accuracy is better than $0.15 \mathrm{~dB}$. A simple method for the calibration of rotating scatterometers (including rotating pencil-beam scatterometer and RFSCAT) using natural extended-area targets is proposed, and then it is verified by the HY-2A scatterometer data and a simulation approach.

To achieve reliable external calibration, an extended land target with spatially and temporally homogeneous backscatter performance needs to be selected. By analyzing the backscatter characteristics of Amazon rainforest, Congo rainforest, Sahara desert, Greenland, and Antarctic ice shells, we find that the radar response over Amazon rainforest and Congo rainforest exhibit spatially and temporally homogeneous and they can be used for the RFSCAT external calibration. Meanwhile, the Greenland and Antarctic ice shells are not suitable as the RFSCAT external calibration targets due to those backscatter anisotropic behaviors. Then the new calibration masks over Amazon rainforest and Congo rainforest are generated and will be used for RFSCAT calibration. As a compromise between the data amount acquired over the calibration targets and the temporal variations in targets' response, a 2-week window is suggested in the calibration procedure of RFSCAT.

The proposed external calibration method calibrates the measurements over different azimuthal intervals $\left(0^{\circ}-360^{\circ}\right)$ to the reference azimuth interval, ensuring consistency among different azimuthal measurements. For RFSCAT onboard CFOSAT, different loading of force of the rotary joint after launch will introduces the azimuthal-dependent insertion loss, which will remain the same amount for a long time. The estimation of loss of the rotary joint will be adjusted after each external calibration operation and they will keep the same values before next calibration. Thus, natural extendedarea targets will be used for the external calibration throughout the whole CFOSAT mission with a period of 1 month. The azimuthal-dependent relative bias of RFSCAT that added in this simulation is assumed to be a sinusoidal function with the maximum magnitude of $0.5 \mathrm{~dB}$. The standard deviation of the $\sigma^{0}$ after calibration is less than $0.06 \mathrm{~dB}$. If the maximum magnitude of the sinusoidal function increases up to $1.0 \mathrm{~dB}$, the standard deviation of the $\sigma^{0}$ after calibration does not change obviously. Thus, the overall calibration error including the internal calibration error, the residual temporal and spatial variations of $\sigma^{0}$ of Amazon and the external calibration error is less than $0.25 \mathrm{~dB}$. The method is also verified by examining the HY-2A scatterometer measurements, which can be treated as a specific case of the rotating antenna scatterometers. For HY-2A scatterometer, the variation of the measurement is due to the pointing errors of the rotating axis. This bias can be estimated with the similar procedure as that of RFSCAT. HY2A scatterometer can be calibrated with a standard deviation less than $0.1 \mathrm{~dB}$. The overall calibration error using tropical rainforest including the model error of rainforest and the external calibration error is less than $0.2 \mathrm{~dB}$.

Due to the lack of the truth $\sigma^{0}$ of the targets, the total end-to-end system gain bias $\sigma_{b}^{0}$ cannot be eliminated by the external calibration using natural extended-area targets. The system gain bias is supposed to be globally uniform; therefore, it can be corrected with some specified calibration targets with good knowledge, e.g., using active/passive ground station or using numerical ocean calibration.

\section{ACKNOWLEDGMENT}

The authors would like to thank D. G. Long and A. Elyouncha for their kind help during study. They also appreciate the Scatterometer Climate Record Pathfinder (SCP), Brigham Young University, for kindly providing the SIR products used in this study and National Satellite Ocean Application Service for providing HY-2A scatterometer L1B data. They also appreciate the five reviewers who helped in improving the article.

\section{REFERENCES}

[1] W. T. Liu, "Progress in scatterometer application," J. Oceanogr., vol. 58, no. 1, pp. 121-136, Feb. 2002.

[2] J. Vogelzang and A. Stoffelen, "NWP model error structure functions obtained from scatterometer winds," IEEE Trans. Geosci. Remote Sens., vol. 50, no. 7, pp. 2525-2533, Jul. 2012.

[3] D. G. Long and G. B. Skouson, "Calibration of spaceborne scatterometers using tropical rain forests," IEEE Trans. Geosci. Remote Sens., vol. 34, no. 2, pp. 413-424, Mar. 1996.

[4] J. Zec, D. G. Long, and W. L. Jones, "NSCAT normalized radar backscattering coefficient biases using homogenous land targets," J. Geophys. Res., vol. 104, no. C5, pp. 11557-11568, May 1999.

[5] A. Elyouncha and X. Neyt, "C-band satellite scatterometers intercalibration,” IEEE Trans. Geosci. Remote Sens., vol. 51, no. 3, pp. 1478-1491, Mar. 2013.

[6] J. J. W. Wilson et al., "Radiometric calibration of the advanced wind scatterometer radar ASCAT carried onboard the METOP-A satellite,' IEEE Trans. Geosci. Remote Sens., vol. 48, no. 8, pp. 3236-3255, Aug. 2010.

[7] A. Stoffelen, "A simple method for calibration of a scatterometer over the ocean," J. Atmos. Ocean. Technol., vol. 16, no. 2, pp. 275-282, Feb. 1999.

[8] R. G. Kennett and F. Li, "Seasat over-land scatterometer data. II. Selection of extended area and land-target sites for the calibration of spaceborne scatterometers," IEEE Trans. Geosci. Remote Sens., vol. 27, no. 6, pp. 779-788, Nov. 1989.

[9] P. K. Yoho and D. G. Long, "Model-based ground station calibration for SeaWinds on QuikSCAT," in Proc. Soc. Photograph. Instrum. Eng. (SPIE), San Diego, CA, USA, 2002, pp. 117-126.

[10] X. Dong, D. Liu, J. Zhu, D. Zhu, and W. Lin, "Some considerations about the in-orbit calibration of spaceborne rotating fan beam radar scatterometer," in Proc. Int. Geosci. Remote Sens. Symp., Vancouver, Canada, 2011, pp. 965-968.

[11] W. Lin and X. Dong, "Design and optimization of a Ku-band rotating, range-gated fanbeam scatterometer," Int. J. Remote Sens., vol. 32, no. 8, pp. 2151-2171, Apr. 2011.

[12] C. Anderson, H. Bonekamp, C. Duff, J. Figa-Saldana, and J. J. W. Wilson, "Analysis of ASCAT ocean backscatter measurement noise," IEEE Trans. Geosci. Remote Sens., vol. 50, no. 7, pp. 2449-2457, Jul. 2012.

[13] M. W. Spencer, C. L. Wu, and D. G. Long, "Improved resolution backscatter measurements with the SeaWinds pencil-beam scatterometer," IEEE Trans. Geosci. Remote Sens., vol. 38, no. 1, pp. 89-104, Jan. 2000. 
[14] M. Portabella and A. Stoffelen, "Scatterometer backscatter uncertainty due to wind variability," IEEE Trans. Geosci. Remote Sens., vol. 44, no. 11, pp. 3356-3362, Nov. 2006.

[15] X. Jiang et al., "The HY-2 satellite and its preliminary assessment," Int. J. Digit. Earth, vol. 5, no. 3, pp. 266-281, May 2012.

[16] D. S. Early and D. G. Long, "Image reconstruction and enhanced resolution imaging from irregular samples," IEEE Trans. Geosci. Remote Sens., vol. 39, no. 2, pp. 291-302, Feb. 2001.

[17] X. Dong, D. Zhu, J. Zhu, and T. Wang, "Progress of development of CFOSAT scatterometer," in Proc. Int. Geosci. Remote Sens. Symp., Munich, Germany, 2012, pp. 237-240.

[18] R. Yun, A. Stoffelen, J. Verspeek, and A. Verhoef, "NWP ocean calibration of Ku-band scatterometers," in Proc. Int. Geosci. Remote Sens. Symp., Munich, Germany, 2012, pp. 2055-2058.

[19] G. B. Skouson, "Calibration of Seasat scatterometer using tropical rainforest," M.S. thesis, Dept. Elect. Comput. Eng., Brigham Young Univ., Provo, UT, USA, 1992.

[20] R. Kumar, S. A. Bhowmick, K. N. Babu, R. Nigam, and A. Sarkar, "Relative calibration using natural terrestrial targets: A preparation towards Oceansat-2 scatterometer," IEEE Trans. Geosci. Remote Sens., vol. 49, no. 6, pp. 2268-2273, Jun. 2011.

[21] D. G. Long and M. R. Drinkwater, "Azimuth variation in microwave scatterometer and radiometer data over Antarctica," IEEE Trans. Geosci. Remote Sens., vol. 38, no. 4, pp. 1857-1870, Jul. 2000.

[22] J. Zhu, X. Dong, W. Lin, and D. Zhu, "Calibration of the Kuband rotating fan-beam scatterometer using land extended-area targets," J. Electron. Inf. Technol., vol. 35, no. 8, pp. 1793-1799, Aug. 2013.

[23] I. J. Birrer, E. M. Bracalente, G. J. Dome, J. Sweet, and G. Berthold, " $\sigma^{0}$ signature of the Amazon rain forest obtained from the Seasat scatterometer," IEEE Trans. Geosci. Remote Sens., vol. 20, no. 1, pp. 11-17, Jan. 1982.

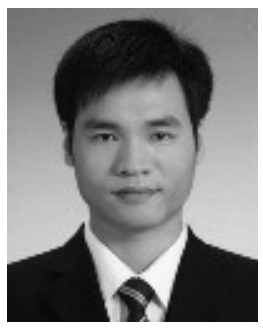

Jintai Zhu was born in Henan Province, China, on March 28, 1987. He received the B.S. degree in engineering from Huazhong University of Science and Technology, Wuhan, China, in 2009, and the $\mathrm{Ph} . \mathrm{D}$. degree in engineering from the University of Chinese Academy of Sciences, Beijing, China, in 2014.

His research interests include calibration of scatterometer.

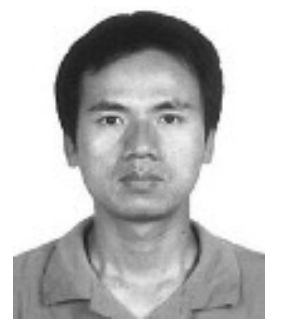

Wenming Lin was born in China, on April 22, 1984. He received the B.Sc. degree in engineering from Wuhan University, Wuhan, China, in 2006, and the Ph.D. degree in engineering from the Center for Space Science and Applied Research, Chinese Academy of Sciences, Beijing, China, in 2011.

$\mathrm{He}$ is currently a Postdoctoral Researcher with the Institute of Marine Sciences (ICM-CSIC), Barcelona, Spain, working on the scatterometer wind quality control.

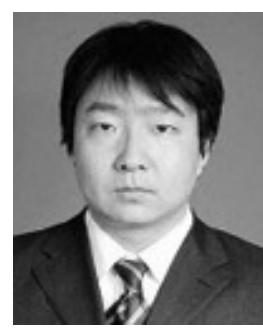

Di Zhu (M'06) was born in Shaanxi Province, China, in 1978. He received the B.S. degree in communication engineering, the M.S. degree in electromaginetic and microwave engineering, in 2001 and 2004, respectively, both from Xi'an Jiaotong University, Xi'an, China, and the Ph.D. degree in computer science from the University of Chinese Academy of Sciences, Beijing, China, in 2008.

From 2008 to 2011, he was an Assitant Professor with the National Space Science Center, Chinese Academy of Sciences, Beijing, China, where he is now an Associate Professor, since 2012. His research interests include spaceborne and airborne remote sensing payload design and signal processing.

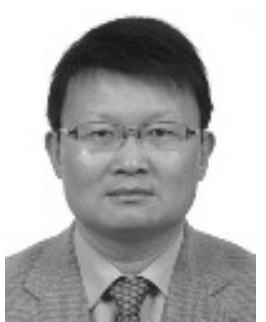

Xiaolong Dong (AM'01-SM'01) was born in Shaanxi Province, China, in 1969. He received the B.E. degree in radio technology and the Ph.D. degree in electromagnetic theory and microwave techniques, in 1991 and 1996, respectively, both from Xi' an Jiaotong University, Xi' an, China.

From 1996 to 1999, he was a Postdoctoral Research Fellow and an Associate Professor with the National Space Science Center, Chinese Academy of Sciences, Beijing, China, and promoted as a Full Professor in 1999. From 2001 to 2003, he visited Duke University, Durham, NC, USA. Since November 2004, he has been a Hundred Talents Professor of the National Space Science Center, Chinese Academy of Sciences, where he is now the Assistant Director-General of the Center and Deputy Director of the CAS Key Laboratory of Microwave Remote Sensing. He has been the Chair of the Microwave Sensors Subgroup of the Working Group on Calibration and Validation of the Committee of Earth Observation Satellites, since 2008. He has been the Principal Investigator of the scatterometers of several Chinese oceanic observation satellites, including the Chinese-French Oceanic Satellite (CFOSAT) and the Haiyang-2 (HY-2) satellite. He is the author of more than 80 journal papers and conference presentation. His research interests include theory and techniques of microwave remote sensing, development of advanced microwave sensors, with emphasis on radar scatterometry for atmospheric, oceanic, and land surface applications. 